\title{
Synthesizing Sum and Difference Patterns with Low Complexity Feeding Network by Sharing Element Excitations
}

\author{
Jafar Ramadhan Mohammed \\ College of Electronics Engineering, Ninevah University, Mosul 41001, Iraq \\ Correspondence should be addressed to Jafar Ramadhan Mohammed; jafarram@yahoo.com
}

Received 12 January 2017; Revised 6 April 2017; Accepted 11 April 2017; Published 20 April 2017

Academic Editor: Herve Aubert

Copyright (C) 2017 Jafar Ramadhan Mohammed. This is an open access article distributed under the Creative Commons Attribution License, which permits unrestricted use, distribution, and reproduction in any medium, provided the original work is properly cited.

\begin{abstract}
In monopulse radar antennas, the synthesizing process of the sum and difference patterns must be fast enough to achieve good tracking of the targets. At the same time, the feed networks of such antennas must be as simple as possible for efficient implementation. To achieve these two goals, an iterative fast Fourier transform (FFT) algorithm is used to synthesize sum and difference patterns with the main focus on obtaining a maximum allowable sharing percentage in the element excitations. The synthesizing process involves iterative calculations of FFT and its inverse transformations; that is, starting from an initial excitation, the successive improved radiation pattern and its corresponding modified element excitations can be found repeatedly until the required radiation pattern is reached. Here, the constraints are incorporated in both the array factor domain and the element excitation domain. By enforcing some constraints on the element excitations during the synthesizing process, the described method provides a significant reduction in the complexity of the feeding network while achieving the required sum and difference patterns. Unlike the standard optimization approaches such as genetic algorithm (GA), the described algorithm performs repeatedly deterministic transformations on the initial field until the prescribed requirements are satisfied. This property makes the proposed synthesizing method converge much faster than GA.
\end{abstract}

\section{Introduction}

Conventional approaches for synthesizing sum and difference patterns require the use of two separate element excitations for one monopulse radar antenna, for example, Taylor excitation [1] for sum pattern formation and Bayliss excitation [2] for difference pattern formation. Thus, these approaches require a feed network of considerable complexity [3-5].

Currently, this drawback of the conventional approaches can be overcome by using subarrays $[6,7]$ or another method known as common element excitations $[8,9]$. Although these approaches allow a significant reduction in the complexity of the feeding network, they rely on the use of an optimization procedure, by either simulated annealing or genetic algorithm. Generally, the optimization algorithms depend on a successive improvement of randomly initialized patterns by applying more or less random variations, which are necessary to overcome local optima. Therefore, the optimization algorithms can be considered as trial-and-error methods and show a rather slow convergence due to the large number of unsuccessful trials [10].
In $[11,12]$, the complexity of the feeding network was simplified to only one attenuator and two phase shifters by controlling the amplitude and phase excitations of the side elements only, while keeping the excitation of the rest of array elements uniform.

This paper introduces a simple and fast algorithm for synthesizing sum and difference patterns with a number of common element excitations for the purpose of reducing the feeding network. For active antenna arrays, the element excitations can be implemented by using a number of digital attenuators and phase shifters. More number of elements results in a complex feeding network. Thus, higher sharing percentage in the element excitations results in lower complexity and cost. By using an iterative fast Fourier transform (FFT) with a specific constraints on the required element excitations and their corresponding radiation patterns, it is possible to reach a good compromise among the sum/difference patterns quality and the complexity of the required feeding networks. Unlike the current optimization approaches, the proposed method may be regarded as a deterministic 
method. Therefore, it delivers results much faster as compared to standard optimization approaches.

\section{Description of the Method}

Consider an array of an even number of isotropic elements $N$ that are equally spaced by $d=\lambda / 2$. For sum beam pattern, the relation between antenna's radiation and its corresponding element excitation can be written as

$$
\operatorname{AF}_{\text {Sum }}(u)=\sum_{n=0}^{N-1} a_{n}^{s} e^{j k n d u}
$$

and, for difference beam pattern, this relation can be given by

$$
\operatorname{AF}_{\text {Diff }}(u)=\sum_{n=0}^{N-1} a_{n}^{d} e^{j k n d u}
$$

where $k=2 \pi / \lambda, \lambda$ representing the wavelength in free space, $u=\sin \theta, \theta$ representing the angle with respect to the direction normal to the array axis, and $a_{n}^{s}$ and $a_{n}^{d}$ are two separate sets of the excitation coefficients belonging to the corresponding sum and difference beam patterns, respectively.

It can be seen from these two equations that the element excitations $a_{n}^{s}$ and $a_{n}^{d}$ and their array patterns $\operatorname{AF}_{\text {Sum }}(u)$ and $\operatorname{AF}_{\text {Diff }}(u)$ are related together through FFT algorithm. In order to apply FFT, the antenna array is assumed to be linear with equally spaced elements. By using these relationships in an iterative manner with some constraints enforcing on the element excitation and sidelobe level, the required goal can be achieved. Specifically, we wish to calculate the coefficients, $a_{n}^{s}$ and $a_{n}^{d}$, so that the corresponding sum and difference beam patterns have a prescribed sidelobe requirements and, at the same time, these two sets have a number of common element excitations. To design such antenna patterns we adopted an iterative fast Fourier transform method [13, 14]. First, we started to calculate the sum and difference patterns assuming some initial values for the coefficients, $a_{n}^{s}$ and $a_{n}^{d}$ (i.e., for $a_{n}^{s}$ we used uniform excitation and for $a_{n}^{d}$ we used odd linear excitation). Since we are using amplitude-only synthesis, the phase of the element excitation is unchanged and thus its value is made to be equal to that of the initial excitation.

As we mentioned earlier, for linear arrays with equally spaced elements, the radiation pattern and its corresponding element excitation are related together by an inverse Fourier transformation. The algorithm starts by updating the sum and difference patterns of the corresponding initial excitations in an iterative manner using 4096-point inverse FFT. During each update, only the sidelobe values that exceed the prescribed sidelobe are modified, and the other sidelobe values are left unchanged. After this modification, a direct 4096point FFT is performed on the adapted sum and difference patterns to get two new sets of the excitation coefficients $a_{n}^{s}$ and $a_{n}^{d}$. Note that the length of vectors $a_{n}^{s}$ and $a_{n}^{d}$ is extended using zero padding. Then, from those 4096 excitation coefficients, only the $N$ samples belonging to the array are retained.

The constraints on the element excitations are performed by replacing the values of a number of the element excitations of the synthesized sum pattern to be equal to that of the synthesized difference pattern.

It is worth mentioning that the constraints are applied to a certain number of element excitations of the corresponding sum pattern (this means that the rest of the element excitations of the synthesized difference pattern remains unchanged). Finally and after applying these element excitation constraints, the fast Fourier transform is performed to get new sum and difference patterns.

\section{Simulation Results}

To validate the effectiveness and the convergence speed of the described method, a number of numerical experiments have been performed on a $2.4 \mathrm{GHz}$ Laptop equipped with a $4 \mathrm{~GB}$ of RAM. In the following examples, the synthesis of equally spaced linear arrays composed of $N=20$ and $N=100$ elements is considered.

As a first example, the iterative FFT algorithm is used to synthesize sum and difference patterns to reach a prescribed sidelobe requirement. In this case, the required sidelobe level of both patterns is chosen to be $-24 \mathrm{~dB}$. Note that, in this example, none of the constraints have been applied to the element excitation, that is, $a_{n}^{s}$ and $a_{n}^{d}$ have no common excitation. The results are shown in Figure 1. This figure also shows the corresponding element excitations for both patterns. Clearly, implementing such antenna with these two separate excitations (i.e., no common excitations) requires a considerable complexity in the feeding network. More importantly, these two excitations tend to be approximately equal at the side elements of the array. This feature can be highly exploited in the proposed method as can be seen in the following examples.

In the second example, both radiation patterns are resynthesized subject to twelve common amplitude excitations (i.e., $60 \%$ of the overall number of array elements is shared for synthesizing sum and difference patterns). The resulting element excitations and the corresponding array patterns are shown in Figure 2. In this case, the element excitations for the difference pattern are kept unchanged (i.e., the same as in Figure 1(b)), while the element excitations corresponding to the sum pattern are modified according to the abovementioned constraints. Accordingly, a new sum pattern with some changes in its sidelobe structure is noticed (see Figure 2(a)). However, the main beam shape of the new sum pattern is maintained undistorted. Although the sidelobe level (SLL) of the resulting sum pattern is higher than the prescribed SLL requirements, the complexity of the feeding network is reduced to more than half with respect to the conventional case (i.e., two separate element excitations for sum and difference pattern with no common excitations).

The sidelobe level (SLL), beam width (BW), taper efficiency, and runtime for these aforementioned two cases (i.e., no common excitations and $60 \%$ common excitations) and for other cases that use different percentage of sharing excitations are reported in Table 1.

It is worth mentioning that the taper efficiency is defined as follows:

$$
\text { Taper Efficiency }=\frac{1}{N} \frac{\left|\sum_{n=1}^{N} a_{n}\right|^{2}}{\sum_{n=1}^{N}\left|a_{n}\right|^{2}},
$$


TABLE 1: Patterns features when $N=20$ and $d=\lambda / 2$ and different percentage of sharing excitations.

\begin{tabular}{|c|c|c|c|c|c|}
\hline & Difference pattern & Sum pattern & \multicolumn{3}{|c|}{ Sum pattern with common excitations constraints } \\
\hline & \multicolumn{2}{|c|}{ No common excitations $0 \%$} & $40 \%$ & $50 \%$ & $60 \%$ \\
\hline SLL $[\mathrm{dB}]$ & -24.0 & -24.0 & -18.0 & -16.32 & -15.32 \\
\hline BW [deg] & 22.8301 & 14.8328 & 13.261 & 13.014 & 12.920 \\
\hline Taper efficiency & 0.5716 & 0.9288 & 0.9580 & 0.9572 & 0.9587 \\
\hline Runtime [second] & 0.012129 & 0.008816 & 0.1209 & 0.1127 & 0.1212 \\
\hline$K[1 / \mathrm{rad}]$ & 0.8573 & - & - & - & - \\
\hline
\end{tabular}

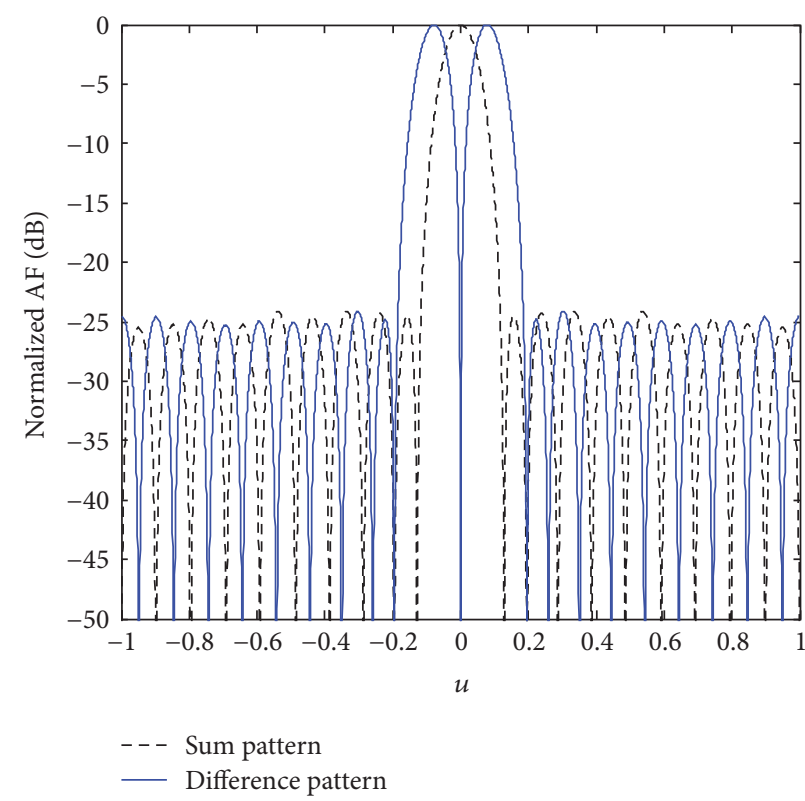

(a)

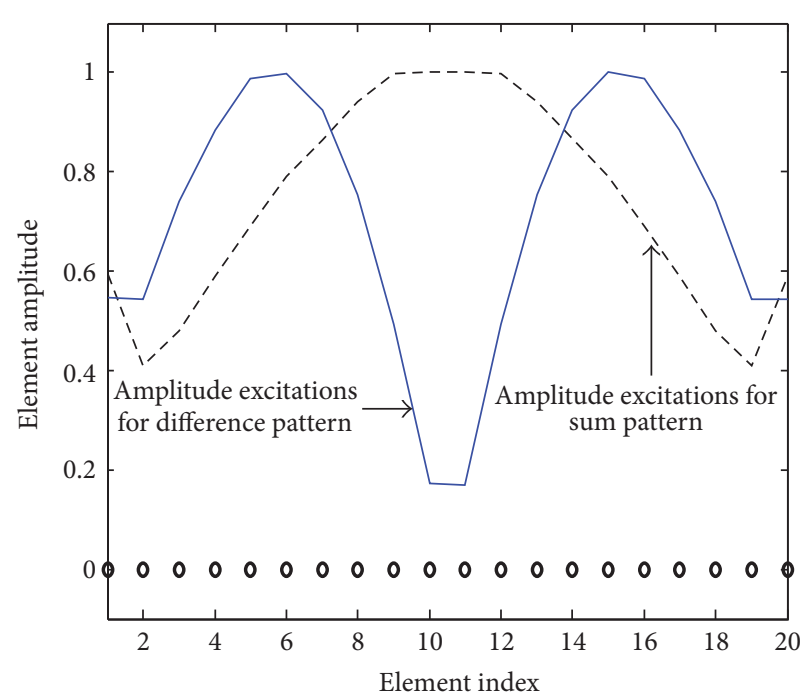

(b)

FIGURE 1: Sum and difference patterns (a) and the corresponding amplitude excitations (b) for the case of no common excitation and $N=20$ elements.

where $a_{n}$ represents the element excitations, whereas the sidelobe level (SLL) is defined as the highest peak level of all side lobes which is usually the closest one to the main beam.

From this table, it can be remarked that the greater the percentage of sharing excitations, the poorer the SLLs of the corresponding sum patterns. Nevertheless, the taper efficiency is slightly increased, and the beam width is significantly decreased when increasing the percentage of sharing excitations.

As mentioned in the previous example, the excitations of the difference pattern were fixed, whereas the excitations of the sum pattern are constraints to $60 \%$ of common excitations. Accordingly, the SLL in the resulting sum pattern was increased. However, this pattern is crucial in the radar applications. Thus, it is desirable to keep its peak sidelobe at low level. By using the proposed method, the excitations of the sum pattern can be easily fixed while those for the difference pattern can be constraints. As with the previous example, $60 \%$ of common excitations are used. The resulting element excitations and the corresponding array patterns are shown in Figure 3. As can be seen, the sum pattern is capable of providing a required SLL which is chosen to be at $-24 \mathrm{~dB}$, while the difference pattern can provide SLL about $8 \mathrm{~dB}$ above the prescribed SLL requirement. However, this sacrifice in the SLL in the difference pattern comes at the good profit in the feeding network.

In the next example, the convergence speed of the iterative FFT algorithm is compared to the standard GA. For fair comparison, it is assumed that the initial excitations or populations for both algorithms are uniform. In addition, the updating processes of these two algorithms are terminated immediately after reaching the allowed number of iterations or prescribed SLL value. In Figure 4, the variations of the maximum SLL, which starts from the initial value of $-13.2 \mathrm{~dB}$ and ends when reaching the required value of $-24 \mathrm{~dB}$, are shown as a function of the iteration number. From this figure, it can be seen that the iterative FFT algorithm is much faster than the solution obtained by GA. Clearly, the described method requires the numerical evaluation of about 4 iterations whereas the GA requires about 100 generations of 


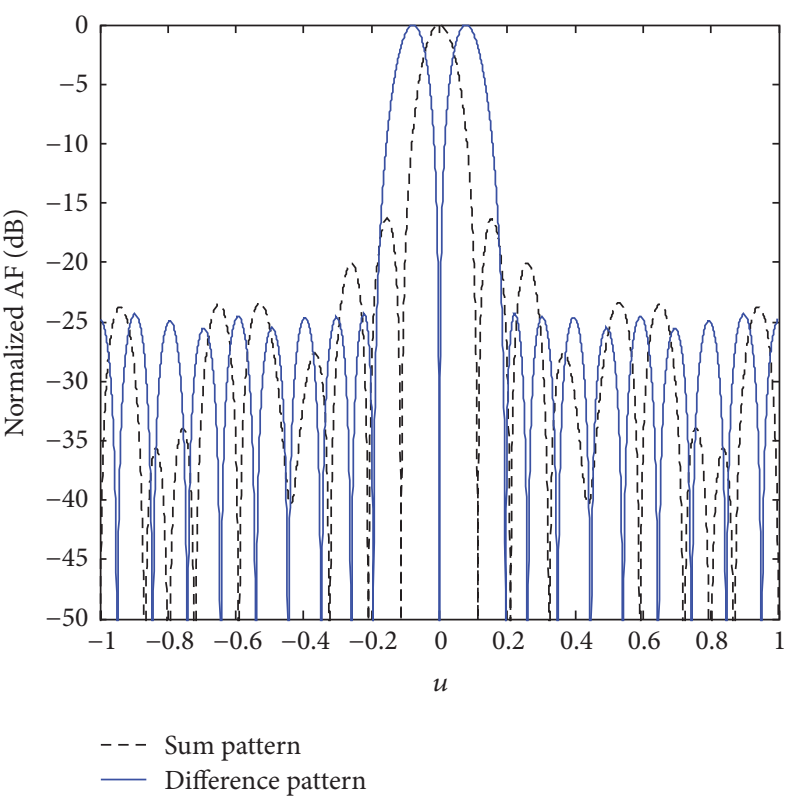

(a)

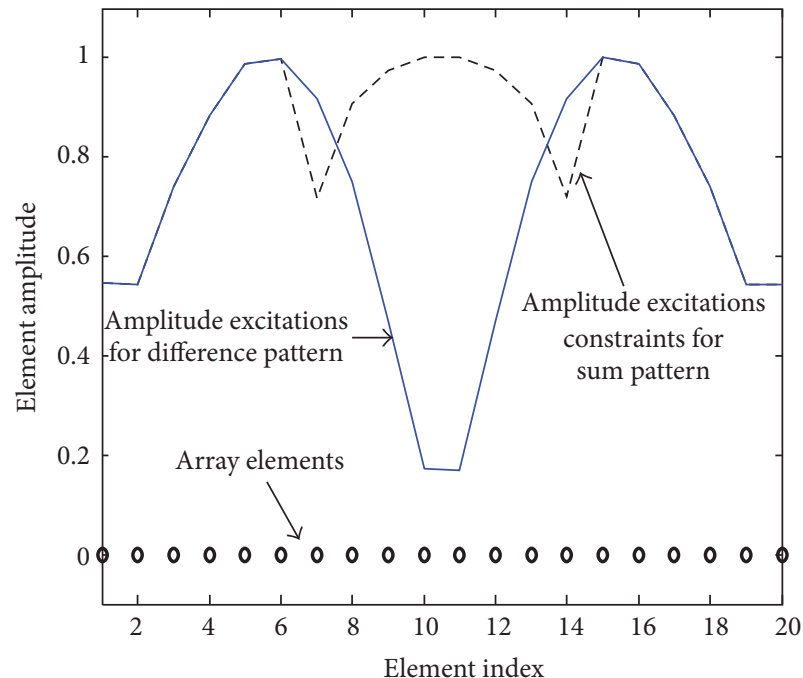

(b)

FIGURE 2: Sum and difference patterns (a) and the corresponding amplitude excitation (b) for the case of fixed difference pattern, $60 \%$ common excitation, and $N=20$ elements.

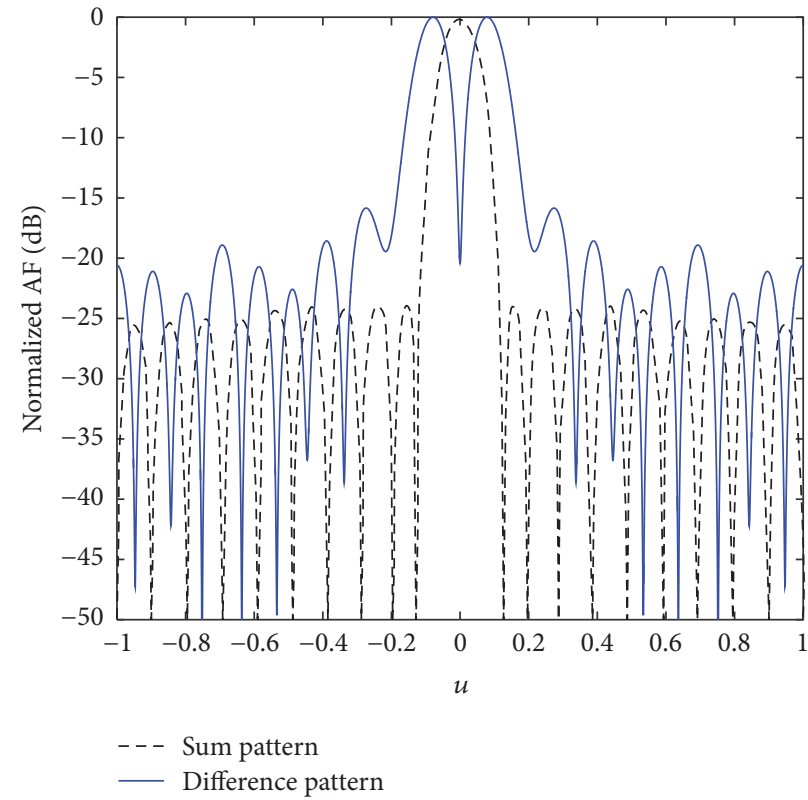

(a)

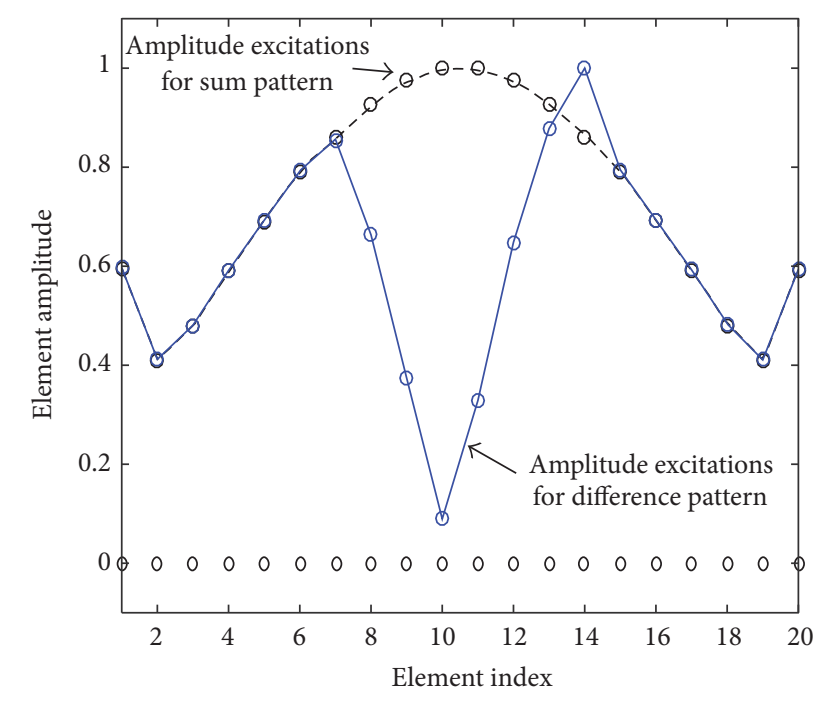

(b)

FIGURE 3: Sum and difference patterns (a) and the corresponding amplitude excitation (b) for the case of fixed sum pattern, $60 \%$ common excitation, and $N=20$ elements.

8 individuals per generation. Here, we used a standard continuous GA, which uses single point crossover and roulette wheel selection.

In the last example, the synthesis of sum and difference patterns with required SLL $=-35 \mathrm{~dB}$ and $N=100$ elements is considered. For no-common-excitation case, the radiation patterns and the corresponding element excitations are shown in Figure 5. Then, both radiation patterns are resynthesized subject to $50 \%$ common excitations. The resulting element excitations and the corresponding array patterns are shown in Figure 6. In this case, the element excitations for the sum pattern are fixed, while those corresponding to 


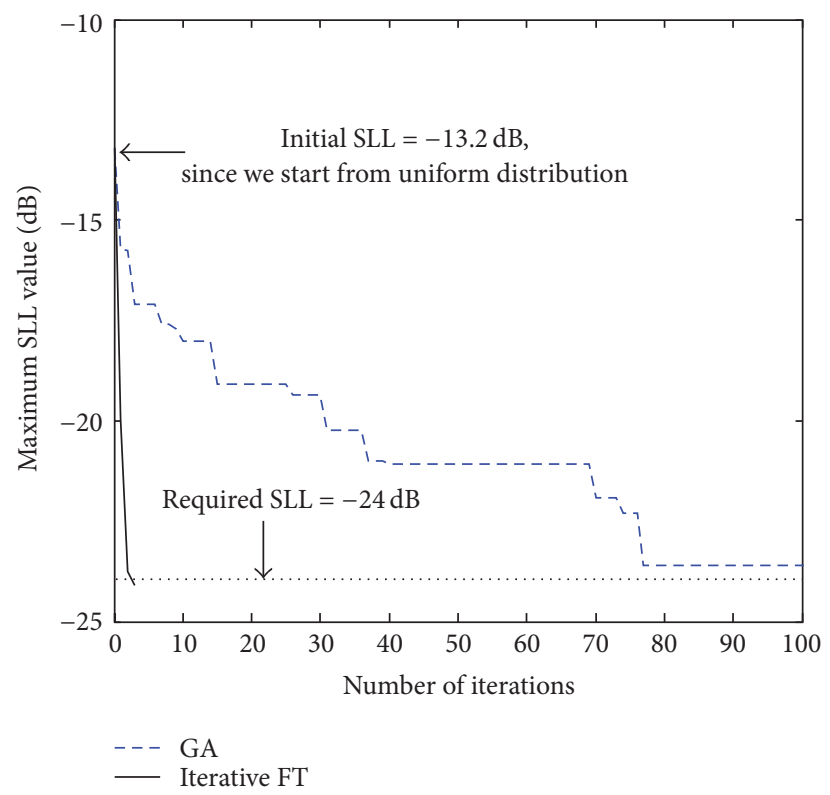

FIGURE 4: Variation of the maximum sidelobe level versus the number of iterations for the sum pattern.

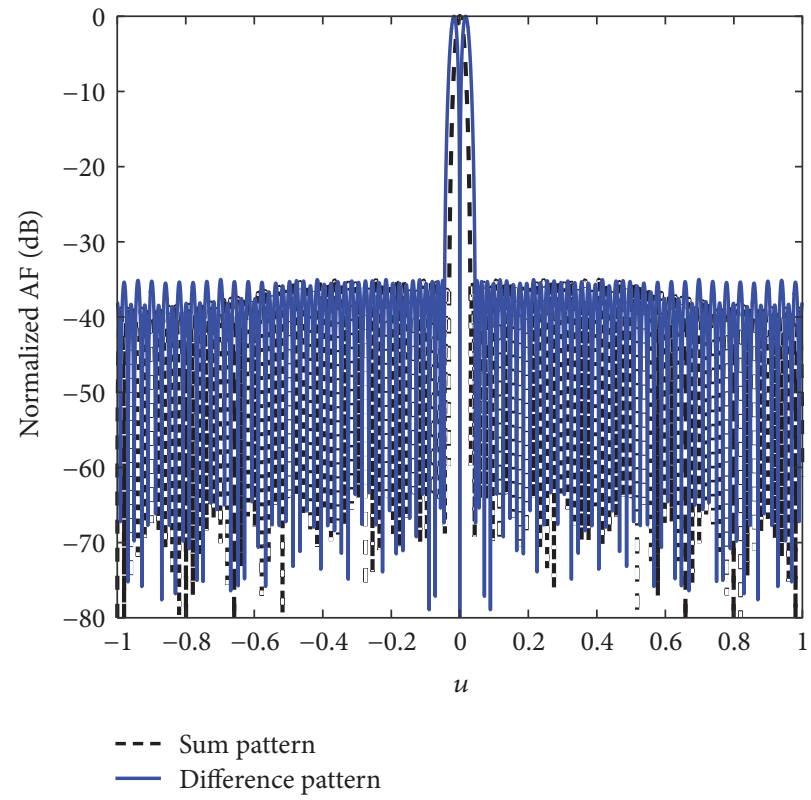

(a)

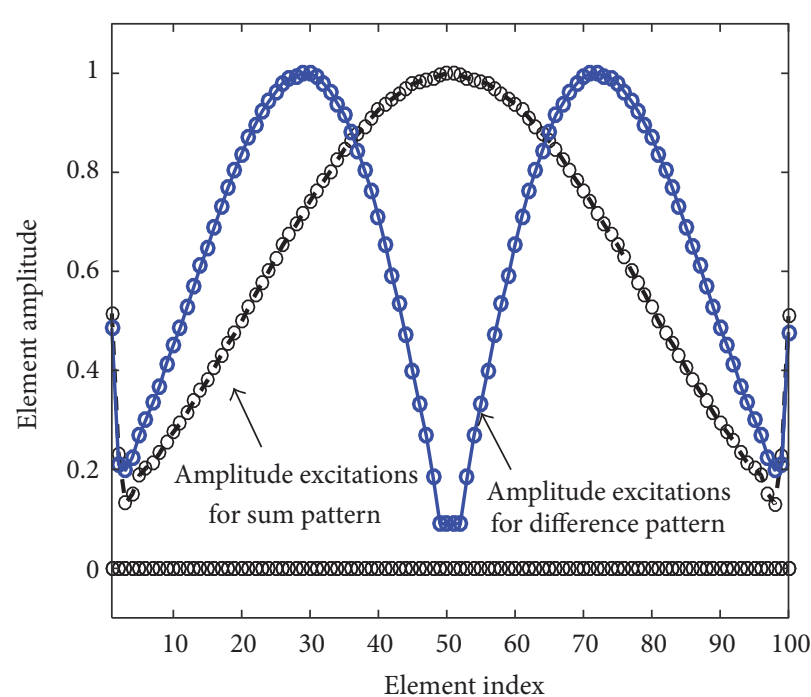

(b)

FIGURE 5: Sum and difference patterns (a) and the corresponding amplitude excitation (b) for the case of no common excitation and $N=100$ elements.

the difference pattern are modified according to the abovementioned constraints. It can be seen that the SLL in the resulting difference pattern was changed from $-35 \mathrm{~dB}$ to $-30.3 \mathrm{~dB}$. Also, the slope near to the boresight is found to be $12.23 \mathrm{~dB}$ /degree. For the sum pattern, the $3 \mathrm{~dB}$ beam width and the taper efficiency are 0.8283 and $1.317^{\circ}$, respectively. Moreover, the runtime required to synthesize the above example was about 0.2718 seconds.

\section{Feeding Network Complexity}

The feeding network complexity and cost are the main challenging issues in practical implementation of the tracking array antennas. Since the amplitude-only control of the element excitation is considered in this study, therefore the complexity and cost in terms of required numbers of amplifiers or attenuators are considered. 


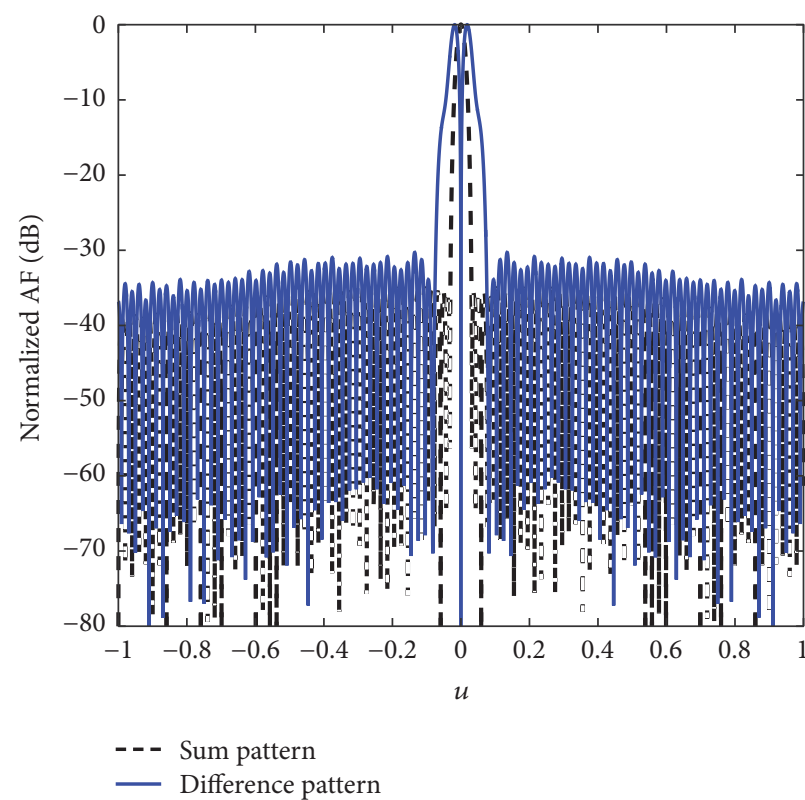

(a)

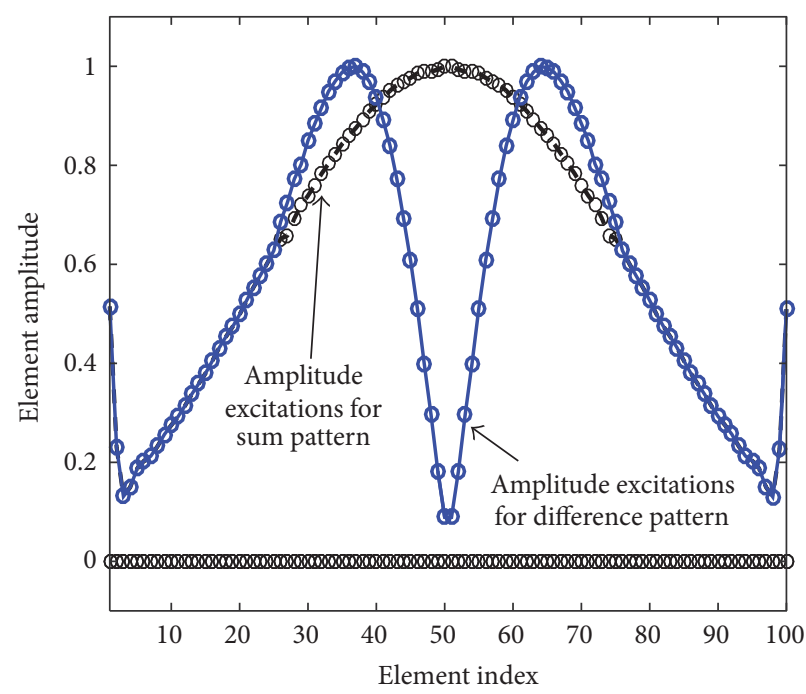

(b)

FIGURE 6: Sum and difference patterns (a) and the corresponding amplitude excitation (b) for the case of fixed sum pattern, 50\% common excitation, and $N=100$ elements.

TABLE 2: Complexity of the feeding network for different cases.

\begin{tabular}{|c|c|c|c|c|c|c|c|c|}
\hline \multirow{3}{*}{ Array patterns } & \multicolumn{8}{|c|}{ Feeding network complexity } \\
\hline & \multicolumn{2}{|c|}{$\begin{array}{c}\text { Separate excitation without } \\
\text { sharing, } 0 \%\end{array}$} & \multicolumn{2}{|c|}{ Sharing percentage, $20 \%$} & \multicolumn{2}{|c|}{ Sharing percentage, $50 \%$} & \multicolumn{2}{|c|}{ Sharing percentage, $80 \%$} \\
\hline & $\begin{array}{l}\text { Number of } \\
\text { attenuators }\end{array}$ & $\begin{array}{l}\text { Peak SLL } \\
(\mathrm{dB})\end{array}$ & $\begin{array}{l}\text { Number of } \\
\text { attenuators }\end{array}$ & $\begin{array}{l}\text { Peak SLL } \\
(\mathrm{dB})\end{array}$ & $\begin{array}{l}\text { Number of } \\
\text { attenuators }\end{array}$ & $\begin{array}{l}\text { Peak SLL } \\
(\mathrm{dB})\end{array}$ & $\begin{array}{l}\text { Number of } \\
\text { attenuators }\end{array}$ & $\begin{array}{l}\text { Peak SLL } \\
(\mathrm{dB})\end{array}$ \\
\hline Sum pattern & $N$ & -24 & $N-N / 4$ & -22 & $N-N / 2$ & -16 & $N-3 N / 4$ & -15 \\
\hline Difference pattern & $N$ & -24 & $N$ & -24 & $N$ & -24 & N & -24 \\
\hline $\begin{array}{l}\text { Total number of } \\
\text { attenuators }\end{array}$ & $2 N$ & - & $2 N-N / 4$ & - & $2 N-N / 2$ & - & $2 N-3 N / 4$ & - \\
\hline
\end{tabular}

The required number of attenuators needed in implementing the feeding network of the sum and difference patterns under different percentage of sharing excitations is reported in Table 2. From this table, it can be seen that, for a tracking antenna with $N=20$ elements, the total number of the required attenuators for synthesizing sum and difference patterns under the case of no sharing excitation is 40 attenuators, while this number is reduced to 30 attenuators under $50 \%$ of sharing excitation and it is further reduced to only 25 attenuators under $75 \%$ of sharing excitation. This reduction in the complexity becomes more significant especially for larger array elements. Moreover, if the array elements possess even symmetry about the center of the monopulse array, the number of attenuators required and the computational time are halved.

\section{Conclusions}

It has been shown that it is possible to generate both sum and difference patterns with a certain number of common excitations. The complexity of the feeding network can be significantly reduced with respect to that case of separate (no sharing) excitations. This reduction comes at the cost of changing the peak SLL in the sum pattern or in the difference pattern. The results show that the greater the percentage of sharing excitations, the poorer the SLLs of the corresponding beam patterns. Thus, the designer needs to trade off between the required sidelobe level and the feed network's complexity.

More importantly, the proposed method converges 100 times faster than the standard GA because the core calculations were based on direct and inverse fast Fourier transforms.

The method can be easily extended to synthesize other patterns such as flat-top beam and pencil beam with common element excitations. Moreover, the principle of the proposed approach may be also extended to the planar arrays having different shapes such as rectangular and circular with uniform element spacing.

\section{Conflicts of Interest}

The author declares that they have no conflicts of interest. 


\section{References}

[1] T. T. Taylor, "Design of circular apertures for narrow beamwidth and low sidelobes," IEEE Transactions on Antennas and Propagation, vol. 8, no. 1, pp. 17-22, 1960.

[2] T. A. Milligan, "Bayliss line-source distribution," in Modern Antenna Design, vol. 7, section 4, pp. 158-161, John Wiley \& Sons, Hoboken, NJ, USA, 2005.

[3] J. R. Mohammed and K. H. Sayidmarie, "A new technique for obtaining wide-angular nulling in the sum and difference patterns of monopulse antenna," IEEE Antennas and Wireless Propagation Letters, vol. 11, pp. 1242-1245, 2012.

[4] J. R. Mohammed, "An alternative method for difference pattern formation in monopulse antenna," Progress in Electromagnetics Research Letters, vol. 42, pp. 45-54, 2013.

[5] K. H. Sayidmarie and J. R. Mohammed, "Performance of a wide angle and wide band nulling method for phased arrays," Progress In Electromagnetics Research M, vol. 33, pp. 239-249, 2013.

[6] M. Alvarez-Folgueiras, J. Rodriguez-Gonzales, and F. AresPena, "Optimal compromise among sum and difference patterns in monopulse antennas: use of subarrays and distributions with common aperture tail," Journal of Electromagnetic Waves and Applications, vol. 23, pp. 2301-2311, 2009.

[7] Q. Zhu, S. Yang, R. Yao, and Z. Nie, "Direction finding using multiple sum and difference patterns in 4D antenna arrays," International Journal of Antennas and Propagation, vol. 2014, Article ID 392895, 12 pages, 2014.

[8] M. Alvarez-Folgueiras, J. Rodriguez-Gonzales, and F. AresPena, "Synthesizing taylor and bayliss linear distributions with common aperture tail," Electronics Letters, vol. 45, no. 11, pp. 1819, 2009.

[9] A. F. Morabito and P. Rocca, "Optimal synthesis of sum and difference patterns with arbitrary sidelobes subject to common excitations constraints," IEEE Antennas and Wireless Propagation Letters, vol. 9, pp. 623-626, 2010.

[10] M. Hacker, G. Stobrawa, and T. Feurer, "Iterative fourier transform algorithm for phase-only pulse shaping," Optics Express, vol. 9, no. 4, pp. 191-199, 2001.

[11] J. R. Mohammed and K. H. Sayidmarie, "Sidelobe cancellation for uniformly excited planar array antennas by controlling the side elements," IEEE Antennas and Wireless Propagation Letters, vol. 13, pp. 987-990, 2014.

[12] J. R. Mohammed and K. H. Sayidmarie, "Null steering method by controlling two elements," IET Microwaves, Antennas \& Propagation, vol. 8, no. 15, pp. 1348-1355, 2014.

[13] W. P. M. N. Keizer, "Fast low-sidelobe synthesis for large planar array antennas utilizing successive fast fourier transforms of the array factor," IEEE Transactions on Antennas and Propagation, vol. 55, no. 3, pp. 715-722, 2007.

[14] J. R. Mohammed, "Phased array antenna with ultra-low sidelobes," Electronics Letters, vol. 49, no. 17, pp. 1055-1056, 2013. 


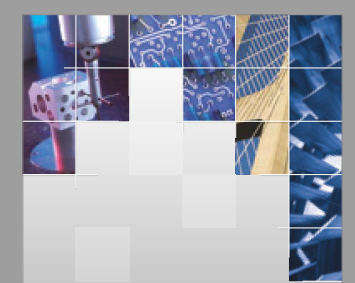

\section{Enfincering}
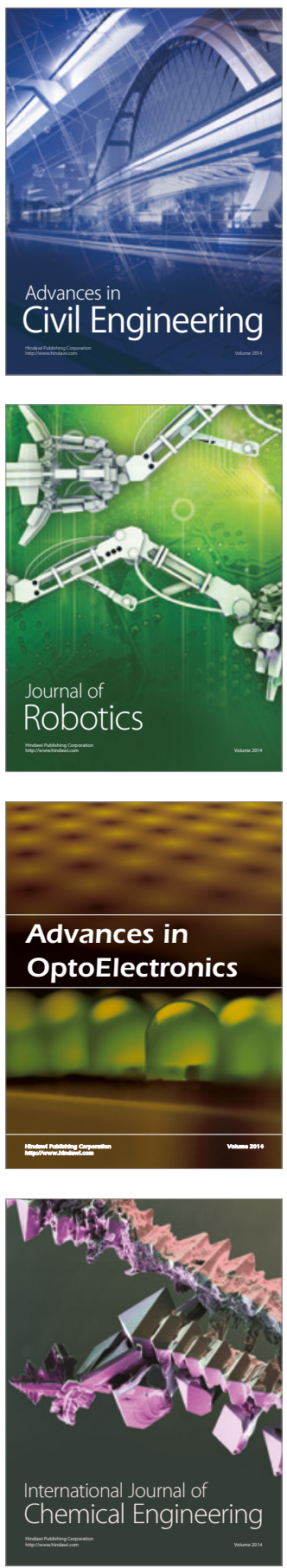

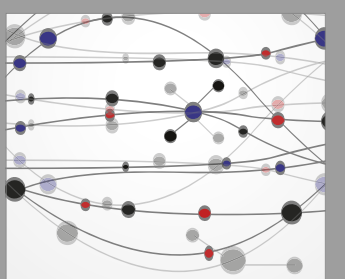

The Scientific World Journal

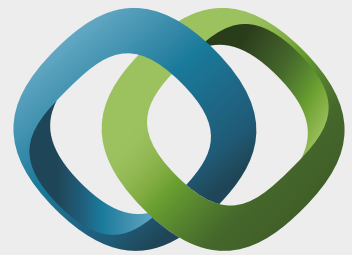

\section{Hindawi}

Submit your manuscripts at

https://www.hindawi.com
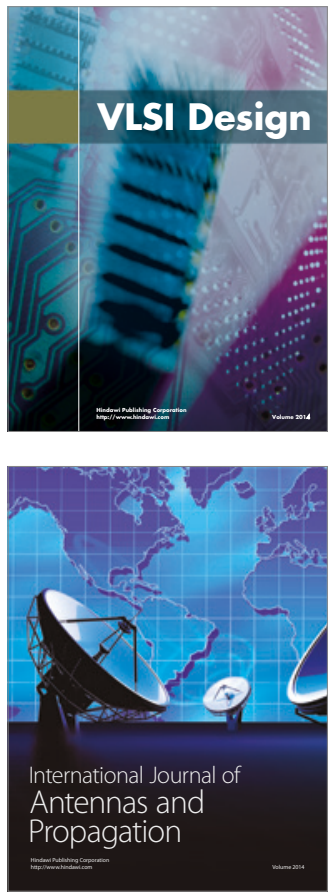

\section{Rotating}

Machinery
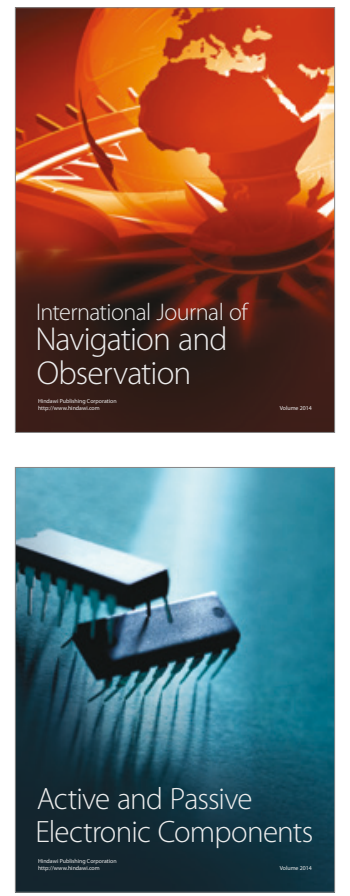
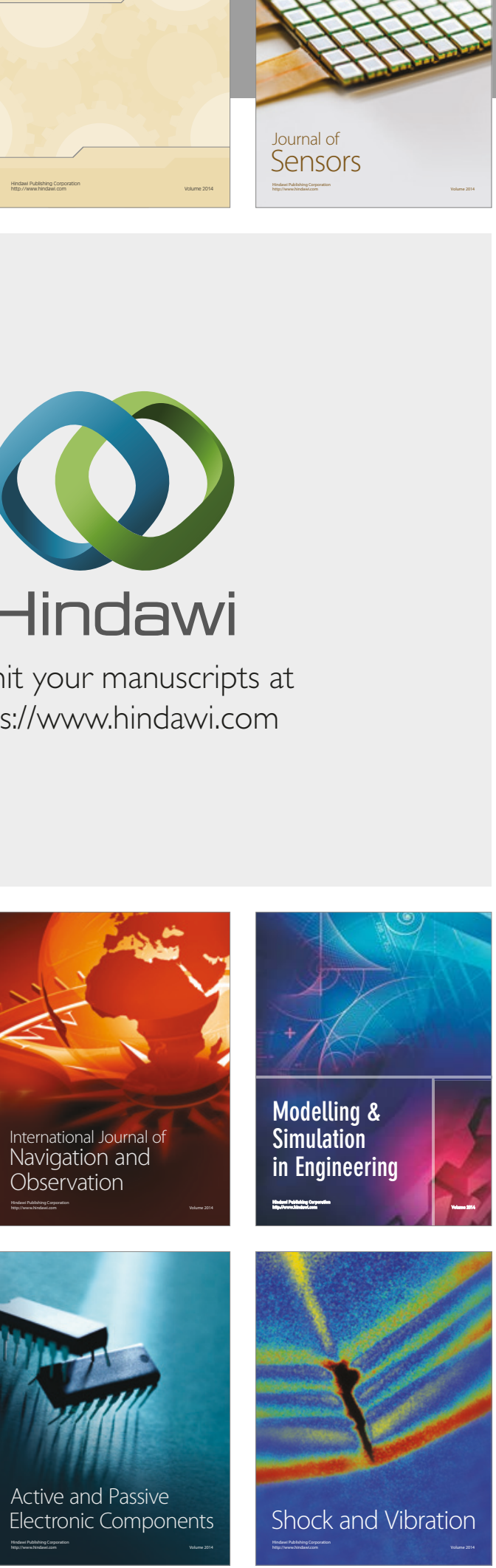
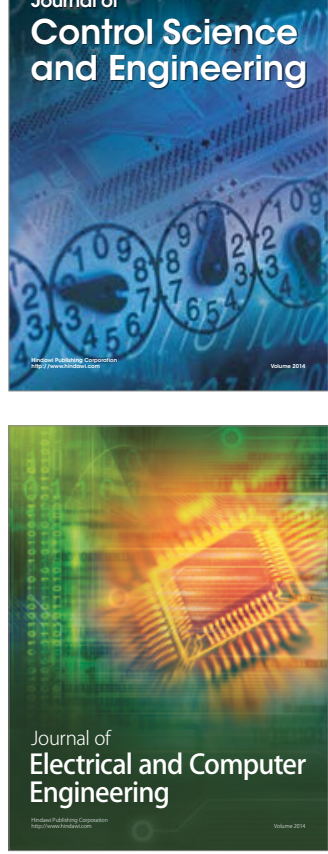

Distributed

Journal of

Control Science

and Engineering
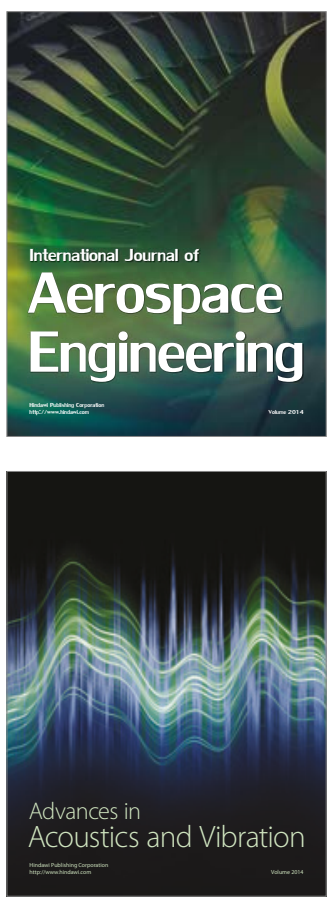

Sensor Networks 